\title{
Costos directos de la artritis reumatoide temprana en el primer año de atención: simulación de tres situaciones clínicas en un hospital universitario de tercer nivel en Colombia
}

\author{
Claudia Mora¹, Andrés González¹, Jorge Díaz², Gerardo Quintana,4 \\ 1 Servicio de Reumatología, Hospital Militar Central, Universidad Militar Nueva Granada, Bogotá, D.C., \\ Colombia \\ 2 Departamento de Farmacia, Facultad de Ciencias. Universidad Nacional de Colombia, Bogotá, D.C., \\ Colombia \\ 3 Departamento de Medicina Interna, Facultad de Medicina, Universidad Nacional de Colombia, Bogotá, \\ D.C., Colombia \\ ${ }^{4}$ Servicio de Reumatología, Hospital Militar Central, Bogotá, D.C., Colombia
}

Introducción. En Colombia se desconoce cuál es la carga derivada de las enfermedades reumatológicas crónicas de forma global y en las diferentes esferas del sistema de salud. La artritis reumatoide es una de las enfermedades más frecuentes en su clase y, actualmente, representa un alto costo para el sistema de salud derivado de la atención de los pacientes.

Objetivo. Determinar los costos médicos directos en pacientes con artritis reumatoide temprana en el primer año de diagnóstico en un hospital universitario de tercer nivel en Colombia.

Materiales y métodos. De acuerdo con la gravedad de la enfermedad medida por el Disease Activity Score 28, se establecieron tres tipos de tratamiento en concordancia con las guías nacionales e internacionales vigentes, que involucran medicamentos modificadores de la enfermedad o inhibidores del factor de necrosis tumoral. Se incluyeron todos los costos directos derivados del cuidado médico, como costos por atención médica especializada, pruebas diagnósticas y medicamentos. El cálculo de los costos se basó en el sistema tarifario del hospital universitario, en el manual tarifario nacional vigente a diciembre de 2007 y en los precios de venta de los fármacos sugeridos al público por la guía Farmaprecios, usada como referencia en las instituciones de salud.

Resultados. En promedio, la atención de la enfermedad leve, moderada y grave cuesta US\$1.689, US\$ 1.805 y US\$23.441, respectivamente. Al utilizar los precios recomendados en Farmaprecios y el manual tarifario nacional, el costo total para cada categoría osciló entre US\$1.418 y US\$ 4.936, US\$ 1.821 y US\$ 7.716, US\$ 31.931 y US\$123.661, respectivamente. El $86 \%$ de estos costos se deriva del costo de los medicamentos, $10 \%$ de los exámenes de laboratorio y $4 \%$ de la atención médica.

Conclusión. El principal elemento de los costos médicos directos en la artritis reumatoide temprana son los costos por medicamentos y se incrementa 40 veces con el uso de la terapia biológica. Deben realizarse estudios de evaluación económica completos para determinar la viabilidad y pertinencia clínica y económica del uso de la terapia biológica en estos pacientes.

Palabras clave: artritis reumatoide, costos y análisis de costos, costo de enfermedad, Colombia.

Financial cost of early rheumatoid arthritis in the first year medical attention: three clinical scenarios.

Introduction. In Colombia, the cost burden of chronic diseases is not well known, either globally or in localized areas of the health system. Rheumatoid arthritis is one of most common chronic diseases, and represents a high cost for the health system.

Objective. The direct medical costs were estimated for rheumatoid arthritis patients in the in the first year of diagnosis at a level 3 university hospital in Colombia. Materials and methods. Three therapy settings for early rheumatoid arthritis patients were established in the first year of diagnosis according to national and international guidelines. Each 


\begin{abstract}
setting included treatment with disease-modifying anti-rheumatic drugs or biologic therapy based on disease severity as measured by Disease Activity Score 28. All direct medical costs were included: specialized medical care, diagnostic tests and drugs. Cost information was obtained from the Central Military Hospital finance department in Bogotá and the national manual of drug prices based on the "Farmaprecios" 2007 guide, a reference in general use by health institutions. Results. The average of cost of medical care in patients with mild, moderate and severe disease was US\$1,689, $\$ 1,805$ and $\$ 23,441$ respectively. The recommended retail prices of the medicines published in "Farmaprecios" was US\$ 1,418, \$1,821 and $\$ 31,931$. When the charges levied by several major health institutions were compared, substantial increases were noted, US\$4,936, \$ 7,716 and \$123,661, respectively. Drug costs represented $86 \%$ of total cost, laboratory costs were $10 \%$ and medical attention was only $4 \%$. Conclusions. Drugs costs were the principal component of the total direct medical cost, and it increased 40 times when a biological therapy is used. Complete economic evaluation studies are necesary to estimate the viability and clinical relevance of biological therapy for early rheumatoid arthritis.
\end{abstract}

Key words: rheumatoid, arthritis; costs and cost analysis, cost of illness, Colombia.

Las enfermedades reumatológicas son entidades crónicas que frecuentemente tienen compromiso sistémico, se manifiestan en la mayoría de casos durante los años de productividad laboral y comprometen negativamente la expectativa de vida. Parte del impacto en salud pública y los costos derivados de estas enfermedades surgen de la discapacidad generada por el compromiso músculo-esquelético o visceral.

Con el conocimiento más profundo de las características fisiopatológicas y el desarrollo de nuevas terapias, las cifras de mortalidad, morbilidad y discapacidad han disminuido de forma importante. Sin embargo, es necesario reconocer que, de la misma forma, el costo derivado de la atención médica, específicamente secundario al uso de nuevos medicamentos, se ha incrementado. Infortunadamente, se desconoce en qué forma el incremento de los costos por medicamentos se ve compensado con la disminución de los costos por menor discapacidad, mejor estado de salud y mejor calidad de vida.

La artritis reumatoide es la enfermedad inflamatoria articular más frecuente, con una prevalencia

\section{Correspondencia:}

Gerardo Quintana, Departamento de Medicina Interna, Universidad Nacional de Colombia, carrera 30 con calle 45, Cuidad Universitaria, Bogotá, D.C., Colombia.

Teléfono: (571) 316-5000, extensiones 15011 y 15012. gquintanal@unal.edu.co

Recibido: 23/05/08; aceptado:11/09/08 mundial de $0,1 \%$ a $1 \%$. Afecta principalmente personas entre los 40 y los 60 años, y su curso crónico con destrucción articular progresiva lleva frecuentemente a discapacidad importante con serias limitaciones en la calidad de vida, la capacidad laboral, la función familiar y las actividades sociales (1-4).

La evaluación económica se está consolidando en muchos países como una herramienta para el análisis de las tecnologías y programas sanitarios. Diferentes empresas consultoras y centros académicos se dedican de forma exclusiva o preponderante a realizar investigación de resultados en salud, que incluye la propia evaluación económica, los estudios de calidad de vida y la utilización de tecnologías y otras técnicas que van más allá de la investigación clínica tradicional. Sin embargo, la utilización sistemática, explícita y transparente de la evaluación económica para la toma de decisiones y la asignación de recursos por parte de los entes aseguradores y reguladores del sistema sanitario, sólo se da en un número relativamente reducido de países (5).

El propósito de un estudio del costo de la enfermedad es identificar y medir todos los costos de una enfermedad en particular, incluyendo los componentes directos, indirectos e intangibles. El resultado expresado en medidas monetarias es una aproximación a la carga total de una enfermedad particularen la sociedad. En Colombia se desconoce cuál es la carga derivada de las 
enfermedades reumatológicas crónicas de forma global y en las diferentes esferas del sistema de salud: prestadores, aseguradores, paciente, familia, industria farmacéutica y entorno social.

Este estudio tiene como objetivo determinar los costos directos derivados de la atención en el primer año de enfermedad en pacientes pertenecientes al régimen de salud de las Fuerzas Militares con artritis reumatoide temprana atendidos en el Hospital Militar Central.

\section{Materiales y métodos}

Este estudio se llevó a cabo en el Hospital Militar Central, centro de atención de IV nivel en Bogotá, cuya área de influencia es de 500.000 pacientes, aproximadamente, y en donde se atiende un promedio de 1.000 consultas mensuales en el servicio de reumatología.

El desarrollo de este estudio se basa en la simulación de situaciones patológicas habituales y se definieron tres categorías de acuerdo con la actividad de la enfermedad, según el Disease Activity Score 28 joint count (DAS-28). El abordaje terapéutico y el seguimiento clínico y paraclínico se plantearon de acuerdo con los parámetros establecidos por la guías de manejo de la artritis reumatoide temprana del Hospital Militar (6), que están en concordancia con las guías nacionales e internacionales vigentes en la actualidad (7-10). Para el diagnóstico de la artritis reumatoide se tuvieron en cuenta los criterios del American College of Rheumatology (11).

La valoración del costo fue elaborada utilizando la perspectiva del pagador. Para la realización del estudio se incluyeron sólo los costos directos derivados del cuidado médico, que se agruparon en las siguientes categorías:

- Costos médicos que incluyeron los costos de la atención ambulatoria, la atención durante la hospitalización y los servicios de profesionales asociados a la salud, como fisioterapeutas y enfermeras.

- Costos de los medicamentos administrados a los pacientes, incluyendo los insumos necesarios para la administración de medicamentos endovenosos.
- Costos de las pruebas diagnósticas, incluyendo los exámenes radiológicos y los estudios inmunológicos.

- Costos de la hospitalización para la realización de procedimientos o aplicación de medicamentos.

El primer grupo incluía pacientes con un valor de DAS-28 menor de 3,2, a quienes se les asignó como tratamiento $7,5 \mathrm{mg}$ de meloxicam cada 3 días y una combinación de dos medicamentos modificadores de la enfermedad, que podía ser metotrexato con hidroxicloroquina, leflunomida con hidroxicloroquina, sulfasalazina con hidroxicloroquina o leflunomida con hidroxicloroquina. La dosis de metotrexato fue de $10 \mathrm{mg}$ semanales por vía oral, la de hidroxicloroquina, de $400 \mathrm{mg}$ diarios, la de leflunomida, $100 \mathrm{mg}$ diarios por tres días y, luego, $20 \mathrm{mg}$ diarios, la de sulfasalazina, $1,5 \mathrm{mg}$ diarios. En caso de recibir metotrexato, se adicionó una dosis semanal de $5 \mathrm{mg}$ de ácido fólico. Este grupo, además, fue valorado por el Servicio de Reumatología cada seis semanas durante tres meses y, luego, cada dos meses para formulación, y para exámenes de seguimiento cada seis semanas por tres meses y, luego, cada cuatro meses.

El segundo grupo lo constituyeron pacientes con un valor de DAS-28 entre 3,2 y 5,1 con controles por Servicio de Reumatología cada seis semanas durante tres meses y, luego, cada dos meses, exámenes de seguimiento cada seis semanas por tres meses y, luego, cada dos meses, y 7,5 mg de meloxicam cada 12 horas. Todos recibieron una combinación de dos medicamentos modificadores de la enfermedad quepodían sermetotrexato con hidroxicloroquina, leflunomida con hidroxicloroquina, sulfasalazina con hidroxicloroquina o leflunomida con hidroxicloroquina y además prednisona, omeprazol y calcio con vitamina $D$. La dosis de metotrexato fue de $15 \mathrm{mg}$ semanales por vía oral, la de hidroxicloroquina, $400 \mathrm{mg}$ día, la de sulfasalazina, $2 \mathrm{~g}$ diarios, la de leflunomida, $100 \mathrm{mg}$ diarios por tres días y, luego, $20 \mathrm{mg}$ diarios, la de prednisona, $10 \mathrm{mg}$ diarios, la de omeprazol, 20 $\mathrm{mg}$ diarios, la de calcio, $600 \mathrm{mg}$ con vitamina $\mathrm{D}$ $400 \mathrm{UI}$. 
El tercer grupo involucró pacientes con un valor de DAS-28 superior a 5,1 con controles por el Servicio de Reumatología cada seis semanas durante tres meses y, luego, cada dos meses, y tratamiento con 7,5 mg de meloxicam cada tres días y $15 \mathrm{mg}$ semanales de metotrexato por vía oral con un inhibidor del factor de necrosis tumoral alfa (anti-FNT). Los exámenes de seguimiento se hicieron cada seis semanas por tres meses y, luego, cada dos meses. Los anti-FNT utilizados incluyeron $40 \mathrm{mg}$ subcutáneos de adalimumab cada dos semanas, $50 \mathrm{mg}$ subcutáneos de etanercept a la semana o infliximab en dosis de $300 \mathrm{mg}$ en bolo aplicados en las semanas 0, 2, 4 y después cada 8 semanas. En el Hospital Militar la aplicación del infliximab es intrahospitalaria, por lo que en el costo de aplicación se incluyó el día de hospitalización.

Los exámenes de laboratorio incluyeron anticitrulinas y factor reumatoideo al inicio, cuadro hemático, VSG, PCR, AST, ALT y fosfatasa alcalina, periódicos, de acuerdo con lo descrito para cada grupo, y radiografía de manos y pies una vez al año. Los pacientes con hidroxicloroquina, además, tuvieron una valoración oftalmológica dos veces al año y campimetría anual; en el grupo 3, además, se incluyó la realización de parcial de orina.

La estimación de costos por medicamentos, exámenes de laboratorio y atención médica, se obtuvo mediante el promedio histórico de los precios en los últimos seis meses en el Hospital Militar Central.

Con el ánimo de dar validez externa a los datos arrojados por este estudio, se aplicó la misma matriz de costos a los tres grupos, reemplazando las tarifas del Hospital Militar Central por tarifas SOAT 2007 (manual tarifario con el cual se determinan la nomenclatura y la clasificación de los procedimientos médicos, quirúrgicos y hospitalarios, según el Decreto 2423 del 31 de diciembre de 1996) para el cálculo de los costos de los exámenes paraclínicos y los costos médicos, ya que éstas son de amplio uso en la mayoría de las empresas de salud del país; también, por los precios sugeridos al público y el promedio del mercado de y para las farmacias independientes de la Guía Farmaprecios No. 99 de noviembre-diciembre de 2007 para el costo por medicamentos.

Todos los costos se calcularon para una duración estándar de tratamiento de 12 meses. Los supuestos del presente modelo incluyen el uso de productos biológicos en forma temprana en la artritis reumatoide en pacientes con enfermedad grave y que los pacientes permanecían estables y no requerían hospitalización por complicaciones derivadas de la enfermedad (sólo en caso de practicarse algún procedimiento diagnóstico o la aplicación de medicamentos). Para el análisis de los resultados, los costos obtenidos en pesos se convirtieron a dólares, según la tasa representativa del mercado a 28 de enero de 2008 (Col\$1.969,95 por dólar).

\section{Resultados}

Los costos para cada grupo se detallan en el cuadro 1. En promedio, la atención de un paciente con enfermedad con actividad leve durante el primer año cuesta US\$ 1.689 (\$3'325.796), con actividad moderada cuesta US\$ 1.805 (\$3'554.193) y con actividad grave cuesta US\$ 23.441 (\$46'155.596).

Del costo total, el $86 \%$ se deriva del costo por medicamentos, el $10 \%$, de laboratorios y sólo el $4 \%$ se origina en la atención médica.

De los costos derivados de los medicamentos, los fármacos modificadores corresponden al $99 \%$ en el primer grupo y, entre $94 \%$ y $98 \%$, en el segundo escenario. El resto corresponde a antiinflamatorios no esteroideos (AINE), ácido fólico y prednisona. En el tercer grupo, con la introducción de los inhibidores del factor de necrosis tumoral, el costo total se incrementó en Col\$ 43 millones y los costos por medicamentos modificadores de la enfermedad, incluso sumado a los costos de otros fármacos, no alcanzan el $1 \%$ del costo total por medicamentos. Además, como se observa en el cuadro 1, según el medicamento modificador de la enfermedad utilizado, hay una variabilidad importante en el costo, con incremento del 100\% cuando se utiliza leflunomida.

Cuando se utilizaron en la matriz de costos los precios de venta al público de los medicamentos 
publicados en Farmaprecios y las tarifas SOAT, los costos totales para el primer grupo oscilaron entre US\$ 1.418 y US\$ 4.936 (Col\$ 2'792.888 a Col\$ 9'718.904), para el segundo, entre US\$ 1.821 y US\$ 7.716 (Col\$ 3'586.640 a Col\$ 15'192.584) y para el tercero, entre US\$ 31.931 y US\$ 123.661 (Col\$ 62'872.950 a Col\$ 243'489.990). El rango del costo está dado por la variabilidad del precio del medicamento según la casa farmacéutica. Estos costos se detallan en el cuadro 2. Igualmente, el principal factor determinante del costo es el precio de los medicamentos, principalmente de los medicamentos modificadores de la enfermedad en los dos primeros grupos y de los inhibidores del factor de necrosis tumoral en el tercer grupo.

\section{Discusión}

Los estudios sobre costo de la enfermedad informan el gasto en el que incurre la sociedad en una entidad patológica en particulary, de esta forma, lo que se ahorraría si ésta se erradicara. Igualmente, identifican los diferentes componentes del costo y la forma en que afecta a cada uno de los actores del sistema de salud. Sin embargo, los estudios sobre el costo de la enfermedad no permiten determinar la eficiencia ni la efectividad de las diferentes tecnologías sanitarias disponibles y no proporcionan información suficiente para realizar evaluaciones económicas completas (12).

Los valores disponibles en la literatura sobre el costo de la atención de la artritis reumatoide

Cuadro 1. Costos médicos directos en el primer año de enfermedad, tarifas del Hospital Militar Central.

\begin{tabular}{|c|c|c|c|c|c|c|c|}
\hline \multirow[b]{2}{*}{ Grupo 1} & \multirow[b]{2}{*}{ MTX-HCQ } & \multirow{2}{*}{$\begin{array}{l}\text { Costo total } \\
\$ 1^{\prime} 786.380 \\
\end{array}$} & \multicolumn{2}{|c|}{$\begin{array}{c}\text { Costo por } \\
\text { medicamentos }\end{array}$} & \multicolumn{2}{|c|}{$\begin{array}{c}\text { Costo por } \\
\text { atención médica }\end{array}$} & \multirow{2}{*}{$\begin{array}{c}\begin{array}{c}\text { Costo por } \\
\text { laboratorios }\end{array} \\
\$ 340.44619,1 \%\end{array}$} \\
\hline & & & $\$ 1^{\prime} 289.934$ & $72,2 \%$ & $\$ 156.000$ & $8,7 \%$ & \\
\hline & LFL-HCQ & $\$ 5^{\prime} 306.829$ & $\$ 4 ' 810.383$ & $90,6 \%$ & $\$ 156.000$ & $2,9 \%$ & $\$ 340.446 \quad 6,4 \%$ \\
\hline & SSZ-HCQ & $\$ 1^{\prime} 952.826$ & $\$ 1^{\prime} 456.380$ & $74,6 \%$ & $\$ 156.000$ & $8,0 \%$ & $\$ 340.44617,4 \%$ \\
\hline & LFL-SSZ & $\$ 4^{\prime} 257.149$ & $\$ 3^{\prime} 821.703$ & $89,8 \%$ & $\$ 117.000$ & $2,7 \%$ & $\$ 318.446 \quad 7,5 \%$ \\
\hline \multirow[t]{4}{*}{ Grupo 2} & MTX-HCQ & $\$ 2 ' 000.286$ & $\$ 1^{\prime} 398.162$ & $69,9 \%$ & $\$ 156.000$ & $7,80 \%$ & $\$ 446.12422,30 \%$ \\
\hline & LFL-HCQ & $\$ 5^{\prime} 492.532$ & $\$ 4 ' 890.408$ & $89,0 \%$ & $\$ 156.000$ & $2,84 \%$ & $\$ 446.124 \quad 8,12 \%$ \\
\hline & SSZ-HCQ & $\$ 2 ' 145.804$ & $\$ 1^{\prime} 543.680$ & $71,9 \%$ & $\$ 156.000$ & $7,27 \%$ & $\$ 446.12420,79 \%$ \\
\hline & LFL-SSZ & $\$ 4^{\prime} 578.152$ & $\$ 4^{\prime} 037.028$ & $88,2 \%$ & $\$ 117.000$ & $2,56 \%$ & $\$ 424.1249,26 \%$ \\
\hline \multirow[t]{3}{*}{ Grupo 3} & Adalimumab & $\$ 42 ' 509.168$ & $\$ 41^{\prime} 874.942$ & $98,5 \%$ & $\$ 117.000$ & $0,3 \%$ & $\$ 517.226$ \\
\hline & Etanercept & $\$ 52^{\prime} 457.228$ & $\$ 51^{\prime} 823.002$ & $98,8 \%$ & $\$ 117.000$ & $0,2 \%$ & $\$ 517.226$ \\
\hline & Infliximab & $\$ 43^{\prime} 500.392$ & $\$ 42^{\prime} 866.166$ & $98,5 \%$ & $\$ 117.000$ & $0,3 \%$ & $\$ 517.226$ \\
\hline
\end{tabular}

MTX: metotrexato; HCQ: hidroxicloroquina; SSZ: sulfasalazina; LFL: leflunomida;

Cuadro 2. Costos médicos directos en el primer año de enfermedad, tarifas SOAT 2007 y guía Farmaprecios.

\begin{tabular}{|c|c|c|c|c|c|c|c|c|c|c|}
\hline \multirow[b]{2}{*}{ Grupo 1} & \multirow[b]{2}{*}{ MTX-HCQ } & \multicolumn{2}{|c|}{ Rango costo total } & \multirow{2}{*}{$\begin{array}{l}\text { Promedio } \\
\text { \$ 3'479.528 }\end{array}$} & \multicolumn{2}{|c|}{$\begin{array}{c}\text { Costo por } \\
\text { medicamentos }\end{array}$} & \multicolumn{2}{|c|}{$\begin{array}{l}\text { Costo por } \\
\text { atención médica }\end{array}$} & \multicolumn{2}{|c|}{$\begin{array}{l}\text { Costo por } \\
\text { laboratorios }\end{array}$} \\
\hline & & $\$ 2^{\prime} 792.888$ & $\$ 4^{\prime} 166.168$ & & $82,8 \%$ & $88,5 \%$ & $5,7 \%$ & $3,8 \%$ & $11,5 \%$ & $7,7 \%$ \\
\hline & LFL-HCQ & $\$ 8^{\prime} 752.460$ & $\$ 9^{\prime} 718.904$ & $\$ 9^{\prime} 235.682$ & $94,6 \%$ & $95,1 \%$ & $1,8 \%$ & $1,6 \%$ & $3,6 \%$ & $3,3 \%$ \\
\hline & SSZ-HCQ & \$ 3'778.544 & $\$ 5^{\prime} 217.464$ & \$ 4'498.004 & $87,3 \%$ & $90,9 \%$ & $4,2 \%$ & $3,0 \%$ & $8,5 \%$ & $6,1 \%$ \\
\hline & LFL-SSZ & $\$ 7^{\prime} 901.304$ & $\$ 8^{\prime} 033.784$ & $\$ 7^{\prime} 967.544$ & $94,5 \%$ & $94,6 \%$ & $1,5 \%$ & $1,5 \%$ & $4,0 \%$ & $4,0 \%$ \\
\hline \multirow[t]{4}{*}{ Grupo 2} & MTX-HCQ & \$ 3'586.640 & $\$ 9^{\prime} 132.800$ & $\$ 6$ '359.720 & $85,0 \%$ & $94,2 \%$ & $4,4 \%$ & $1,7 \%$ & $10,57 \%$ & $4,1 \%$ \\
\hline & LFL-HCQ & $\$ 9^{\prime} 613.820$ & $\$ 15^{\prime} 192.584$ & $\$ 12^{\prime} 403.202$ & $94,5 \%$ & $96,5 \%$ & $1,6 \%$ & $1,0 \%$ & $3,91 \%$ & $2,5 \%$ \\
\hline & SSZ-HCQ & \$ 4'639.904 & $\$ 10^{\prime} 222.424$ & $\$ 7^{\prime} 431.164$ & $88,5 \%$ & $94,8 \%$ & $3,4 \%$ & $1,5 \%$ & $8,15 \%$ & $3,7 \%$ \\
\hline & LFL-SSZ & \$ 9'476.184 & $\$ 14^{\prime} 390.184$ & $\$ 11 ' 933.184$ & $94,8 \%$ & $96,6 \%$ & $1,2 \%$ & $0,8 \%$ & $3,97 \%$ & $2,6 \%$ \\
\hline \multirow[t]{3}{*}{ Grupo 3} & Adalimumab & $\$ 69^{\prime} 147.750$ & $\$ 137^{\prime} 793.318$ & $\$ 103^{\prime} 470.534$ & $99,2 \%$ & $99,6 \%$ & $0,2 \%$ & $0,1 \%$ & $0,7 \%$ & $0,3 \%$ \\
\hline & Etanercept & $\$ 62^{\prime} 872.950$ & $\$ 243^{\prime} 489.990$ & $\$ 153^{\prime} 181.470$ & $99,1 \%$ & $99,8 \%$ & $0,2 \%$ & $0,0 \%$ & $0,7 \%$ & $0,2 \%$ \\
\hline & Infliximab & $\$ 69^{\prime} 780.450$ & $\$ 72^{\prime} 704.130$ & $\$ 71^{\prime} 242.290$ & $99,2 \%$ & $99,2 \%$ & $0,2 \%$ & $0,2 \%$ & $0,7 \%$ & $0,6 \%$ \\
\hline
\end{tabular}

MTX: metotrexato; HCQ: hidroxicloroquina; SSZ: sulfasalazina; LFL: leflunomida 
desde las diferentes perspectivas y componentes del mismo, son aproximaciones y las variaciones entre países o ciudades, entre sistemas de salud y localizaciones geográficas hacen difícil su comparación.

En la comunidad europea (13) los costos directos por paciente por año se han estimado entre $€ 1.812$ y $€ 11.792 \mathrm{y}$, los indirectos, entre €1.260 y $€ 37.994$. Se ha calculado que los costos directos abarcan de un cuarto hasta un poco más de la mitad de los costos totales. Los costos asociados con el cuidado hospitalario contribuyen en $75 \%$ a los costos directos, en comparación con sólo $20 \%$ por medicamentos, aunque pueden ocurrir variaciones derivadas del monitoreo del medicamento y por efectos adversos. Las visitas médicas constituyen el $20 \%$ de los costos directos. Los costos indirectos contribuyen al $80 \%$ del exceso del costo relacionado con la artritis reumatoide. La discapacidad tiene un impacto mayor en los costos.

En Estados Unidos para el 2001 (14), el promedio del costo directo anual total era de US\$ $9,519,66 \%$ correspondiente a los costos por medicamentos y $17 \%$ a los costos hospitalarios. Para los pacientes con terapia biológica, el costo total fue de US\$19,016 por año, frente a US\$ 6,164 para aquéllos con terapia convencional. La discapacidad incrementó los costos directos en US\$ 5,022 por año. Para el 2005 (15), un año de terapia para artritris reumatoide con dosis estándar de etanercept y adalimumab era de US $\$ 15,680$, aproximadamente, y el de infliximab osciló entre US\$10,450 y US\$ 18,110 con una diferencia importante respecto al costo del uso de metotrexato genérico, en dosis semanal de $17,5 \mathrm{mg}$, que fue de US\$ 500 .

En el Reino Unido (16) se publicó que los costos totales oscilaron entre US\$ 5.720 y US\$ 5.822 , los medicamentos contribuyeron con $8 \%$ a $24 \%$ de los costos, la atención médica con $8 \%$ a $21 \%$ y las hospitalizaciones con $17 \%$ a $88 \%$.

En el presente estudio los resultados indican que, en promedio, los costos directos en artritis reumatoide temprana son de US\$ 1.689 a US\$ 1.805 con tratamiento convencional con medicamentos modificadores de la enfermedad y de US\$ 23.441 dólares al utilizar anti-FNT durante el primer año de tratamiento.

En Colombia se realizó un estudio similar en el 2004 en Medellín (17), y se encontró que el costo total directo de la artritis reumatoide, considerando el metotrexato como medicamento de primera intención, fue de US\$938 en el primer año, de los cuales, las medicaciones representan más del $50 \%$, seguido por los costos de las intervenciones de laboratorio; mientras que los costos de atención médica representan el porcentaje más bajo $(5,6 \%)$. Cuando al modelo se le agrega leflunomida, la carga del costo de los medicamentos se incrementa a $71 \%$. Los datos aportados por este estudio no son comparables con el actual, debido a la variabilidad en las tarifas según el pagador, la utilización de dosis bajas de metotrexato y la inclusión solamente de tarifas SOAT en los costos de atención médica especializada y no de tarifas derivadas de la atención particular.

Los resultados del estudio actual no son comparables con los realizados en otras poblaciones pues, como se mencionó, hay una amplia variabilidad entre las diferentes regiones geográficas que limitan la comparación y, además, la mayoría de los estudios sobre costos de la enfermedad en artritis reumatoide incluyen pacientes cuya enfermedad en promedio tiene 15 años de evolución, en los cuales los reemplazos articulares, las hospitalizaciones por actividad de la enfermedad y por el manejo de las complicaciones crónicas de la enfermedad corresponden a un porcentaje importante de los costos totales de atención al año.

En el presente estudio es evidente que el principal elemento del costo total de la atención en los pacientes con artritis reumatoide temprana es el costo por medicamentos y con el uso de la terapia biológica se incrementa 40 veces. Sin embargo, en este estudio sólo se tuvieron en cuenta los costos directos y se desconoce cuál es la contribución de los costos directos no derivados de la atención médica, de los costos indirectos y de los intangibles. Aparentemente, desde la perspectiva del pagador el costo de los medicamentos modificadores de la enfermedad, 
como la leflunomida, o de la introducción de la terapia biológica en el primer año de enfermedad no sería justificable, pero con la metodología actual no es posible comprender si los costos derivados de las hospitalizaciones por actividad de la enfermedad disminuirían o cómo se modificaría el costo por discapacidad o por compromiso en la calidad de vida con el uso de estas terapias al alcanzar un mejor control de la enfermedad. Además, la construcción de la matriz de costos se hizo desde la perspectiva del pagador y los valores se tomaron a partir de un régimen especial en salud, como es el sistema de salud de las Fuerzas Militares, lo que limita la validez externa de los resultados; el promedio de los costos de medicamentos por los precios de venta al público puede estar por encima de los precios de venta a empresas promotoras de salud (EPS).

Este estudio, además, excluyó variables como sexo, edad, nivel educativo y enfermedades concomitantes que, según estudios previos en cohortes, pueden aumentar los costos directos.

No se conocen datos certeros de la prevalencia de la enfermedad en Colombia, pero en Latinoamérica es de $0,5 \%$ (18). Si se extrapola esta cifra a Colombia, 225.000 personas requerirían atención por artritis reumatoide. El Sistema General de Seguridad Social colombiano definió el monto necesario para cubrir en integridad los contenidos del Plan Obligatorio de Salud (POS) para cada usuario como unidad de pago por capitación (UPC), cuyo valor actual es de \$ $386.881,20$ anuales para el régimen contributivo y de \$215.712,00 anuales para el régimen subsidiado. El costo de la atención de un paciente con artritis reumatoide temprana, teniendo en cuenta únicamente costos directos, es 8 a 119,3 veces superior al valor de la UPC, lo que claramente resulta en recursos monetarios disponibles insuficientes. Además, los medicamentos como la hidroxicloroquina, la leflunomida y los antiFNT no están incluidos en el POS.

Teniendo en cuenta la prevalencia de la enfermedad, la carga que representa para el sistema de salud y para la sociedad, y la evidencia de que la diferencia en costos según la gravedad de la enfermedad es importante, es imperativo el desarrollo de estudios de prevalencia, incidencia, efectividad clínica y de evaluación económica en la artritis reumatoide en Colombia. La evidencia mundial sugiere que se justifica un tratamiento agresivo de forma temprana, que busca mantener la remisión clínica y disminuir la progresión de la enfermedad y el tratamiento de complicaciones, mejorar la calidad de vida de los pacientes y, posiblemente, de forma paralela, disminuir la carga económica del sistema de salud.

Ésta es una aproximación general en la evaluación económica en reumatología y una invitación al diseño de estudios completos de evaluación económica (costo-minimización, costo-efectividad, costo-utilidad, costo-beneficio) que permitan, una vez reconocida la carga de enfermedad, dirigir la toma de decisiones en salud y el destino de los recursos, para garantizar el máximo beneficio para el paciente con el menor impacto para el sistema de salud.

\section{Conflictos de intereses}

Los autores no declaran ningún conflicto de interés.

\section{Financiación}

Autofinanciación.

\section{Referencias}

1. Guzmán R, Restrepo J. Artritis reumatoide temprana. Rev Colomb Reumatol. 2002;9:171-5.

2. Choy EH, Panayi GS. Cytokine pathways, and joint inflammation in rheumatoid arthritis. $\mathrm{N}$ Engl $\mathrm{J}$ Med. 2001;344:907-16.

3. Merkesdal S, Ruof J, Schoffski O, Bernitt K, Zeidler H, Mau W. Indirect medical cost in early rheumatoid arthritis. Arthritis Rheum. 2001;44:528-34.

4. Combe B. Early rheumatoid arthritis: strategies for prevention and management. Best Pract Res Clin Rheumatol. 2007:21:27-42.

5. Rovira J. Evaluación económica en salud: de la investigación a la toma de decisiones. Rev Esp Salud Pública. 2004;78:293-5.

6. Mora C, González A, Quintana G. Guía de manejo artritis reumatoide temprana en un Hospital Universitario de Colombia. Rev Colomb Reumatol. 2008;15:79-91.

7. Combe B, Landewe R, Lukas C, Bolosiu HD, Breedveld $F$, Dougados M, et al. EULAR recommendations for the 
management of early arthritis: report of a task force of the European Standing Committee for International Clinical Studies Including Therapeutics (ESCISIT). Ann Rheum Dis. 2007;66:34-45.

8. Luqmani R, Hennell S, Estrach C, Birrell F, Bosworth A, Davenport G, et al. British Society for Rheumatology and British Health Professionals in Rheumatology Guideline for the Management of Rheumatoid Arthritis (the first 2 years). Rheumatology. 2006;45:1167-9.

9. Vinicio C, Chalem P, Londoño L, Restrepo J, Iglesias $\mathbf{A}$, Vélez $\mathbf{P}$, et al. Primer consenso colombiano sobre el tratamiento de la artritis reumatoide temprana. Rev Col Reumatol. 2002;9:323-31.

10. Loët X, Berthelot J M, Cantagrel A, Combe B, De Bandt M, Fautrel B, et al. Clinical practice decision tree for the choice of the first disease modifying antirheumatic drug for very early rheumatoid arthritis: a 2004 proposal of the French Society of Rheumatology. Ann Rheum Dis. 2006;65:45-50.

11. Arnett FC, Edworthy SM, Bloch DA, McShane DJ, Fries JF, Cooper NS, et al. The American Rheumatism Association 1987 revised criteria for the classification of rheumatoid arthritis. Arthritis Rheum. 1988;31:315- 24.
12. Byford S, Torgerson D, Raftery J. Economic note: Cost of illness studies. BMJ. 2000;320;1335.

13. Rat AC, Boissier MC. Rheumatoid arthritis: direct and indirect costs. Joint Bone Spine. 2004;71:518-24.

14. Michaud K, Messer J, Choi H, Wolfe F. Direct medical costs and their predictors in patients with rheumatoid arthritis a three-year study of 7,527 patients. Arthritis Rheum. 2003;48:2750-62.

15. Kavanaugh A. The pharmacoeconomics of newer therapeutics for rheumatic diseases. Rheum Dis Clin North Am. 2006;32:45-56.

16. Cooper NJ. Economic burden of rheumatoid arthritis: a systematic review. Rheumatology. 2000;39:28-33.

17. Pineda-Tamayo R, Arcila G, Restrepo P, Tobón GJ, Camargo JF, Anaya JM. Costos médicos directos de la artritis reumatoide temprana. Rev Colomb Reumatol. 2004;11:89-96.

18. Delgado-Vega A, Martín J, Granados J, Anaya JM. Epidemiología genética de la artritis reumatoide: ¿qué esperar de América Latina? Biomédica. 2006;26:562-84. 